\title{
A Triband Planar Inverted-F Antenna with Quadratic Koch Fractal Shaped Slit Along with a Shorted Parasitic Strip
}

\author{
Dipak Kumar' ${ }^{1}$ Deblina Chowdhury ${ }^{1}$ and Rowdra Ghatak ${ }^{1}$ \\ ${ }^{1}$ Microwave and Antenna Research Laboratory, Electronics and Communication Engineering Department, National Institute of \\ Technology Durgapur, Durgapur-713209, West Bengal, India \\ *Deblina Chowdhury, E-mail: chowdhury. deblina@yahoo.com
}

\begin{abstract}
In this paper, a novel compact tri-band planar inverted-F antenna (PIFA) for mobile communication application is proposed. The antenna is capable to cover GSM $900 \mathrm{MHz}$, DCS $1.8 \mathrm{GHz}$ and WLAN (IEEE 802.11b) $2.45 \mathrm{GHz}$ bands. The proposed PIFA is composed of a quadratic Koch shape slit and a parasitic strip. The PIFA with the fractal shaped slit contributes to the first and second resonance while the shorted strip brings forth the third band. The impedance bandwidths of $84 \mathrm{MHz}, 132 \mathrm{MHz}$ and $81 \mathrm{MHz}$ for GSM 900, DCS 1800 and WLAN (IEEE 802.11b) 2450, respectively are achieved. A realized gain of $2.44 \mathrm{dBi}, 4.48$ $\mathrm{dBi}$ and $3.86 \mathrm{dBi}$ is obtained at $0.9 \mathrm{GHz}, 1.8 \mathrm{GHz}$ and 2.45 $\mathrm{GHz}$, respectively. The proposed antenna is fabricated and $\left|\mathrm{S}_{11}\right| \mathrm{dB}$ is measured. Reasonable agreement between simulated results as well as measured results is obtained.
\end{abstract}

\section{Introduction}

An indispensable component in a wireless transceiver is an antenna. The shrinking of available antenna placement space has ushered into a new paradigm of transceiver design because of the advent of smart phones, where the designer puts in additional features which are based on different frequency bands. Planar Inverted-F antenna (PIFA) has been a preferred choice of transceiver designers as an internal antenna for mobile handset due to its low profile, small size, built-in structure, ease of fabrication and low manufacturing cost. PIFA antenna structure is a modified form of the conventional quarter wavelength $(\lambda / 4)$ monopole antenna.

Mobile phone internal antenna, which is integrated in the handset, offers certain advantages in comparison to conventional external antennas like a monopole or helix, it is not vulnerable to damages and can offer reduced SAR [1]. There have been a number of PIFA designs that describe different configurations to achieve multiple band operation [2]-[14]. Multiband PIFA was obtained by implementing various shapes and sizes of slot and slit in the radiating patch [2]-[9]. Dual-band PIFAs were introduced with various techniques like, using a capacitive feed and a capacitive load [3], embedding radiating patch with different shapes of slot and slit like U-slot, V-slot, L-slit, Tshaped slit [4]-[9]. In [10], Hilbert curve PIFA geometry was employed for overall size reduction, but matching obtained at higher bands has not been impressive. Adding parasitic patch technique also facilitates additional resonance modes in [11]. Antenna designers also developed the PIFA geometry with more compactness using slots and slits for tri-band application [12]-[14].In [15] another compact structure has been proposed, where a three branch top patch has been used to resonate three frequency bands. A multiband PIFA has been proposed where, instead of top patchgridded ground plane structure with overlapping cells are used to achieve multiband operation [16].

In this paper, a simple and compact PIFA covering three frequency bands: GSM $(900 \mathrm{MHz})$, DCS $(1.8 \mathrm{GHz})$ and WLAN (IEEE 802.11b) $2.45 \mathrm{GHz}$ is proposed invoking a fractal shaped slit on the radiating patch as well as adding a parasitic strip. A quadratic Koch shaped slit is implemented on the radiating patch to get the dual-band resonance frequency as well as to reduce the patch size. The total volume of antenna is $35.65 \times 20 \times 8 \mathrm{~mm}^{3}$. Rest of the paper is described as follows. Step by step implementation of triband PIFA is given in section 2. This is followed by discussion on results in section 3. Concluding remarks are given in step 4.

\section{Antenna design and parametric studies}

The geometry of the proposed antenna is shown in Fig. 1. It illustrates the antenna design that comprises three design steps which results in a PIFA having the ability of radiating at three different bands. The step by step implementation of the PIFA is elucidated in three sub-sections. The radiating part is consiered to be made of copper of thickness $0.25 \mathrm{~mm}$ and the ground is realized on a FR-4 substrate of height 1.6 $\mathrm{mm}$, having copper deposit on one side. It has a length of $100 \mathrm{~mm}$, and width of $45 \mathrm{~mm}$. The antenna is fed with a 50 $\Omega$ SMA connector. For all electromagnetic simulation CST Microwave Studio ${ }^{\mathrm{TM}}$ is used. 


\subsection{Initial PIFA design}

For the conventional PIFA antenna, the initial radiator dimension is obtained at the desired first resonance frequency of $900 \mathrm{MHz}$. This is done by using the equation given in (1) [4].

$$
f=\frac{c}{4 \sqrt{\varepsilon_{r}\left(L_{p}+W_{p}\right)}}
$$

In (1),$f$ is the resonant frequency, $c$ is the velocity of light in free space, $\varepsilon_{r}$ is the dielectric constant of the medium in between top patch and ground, here we use air $\left(\varepsilon_{r}=1\right) . L_{p}$ and $W_{p}$ are the length and width of the radiating patch. The initial PIFA patch dimension is calculated to be $45 \mathrm{~mm}$ in length, and $21 \mathrm{~mm}$ in width,with an antenna height of $8 \mathrm{~mm}$. The total length of the current path, i.e, the total length ( $L_{\text {Total }}$ $\left.=L_{p}+W_{p}+h\right)$ of the radiating patch, which is $74 \mathrm{~mm}$, determines the approximate resonant frequency of the antenna. Where $h$ is the height of antenna. The simulated $\left|\mathrm{S}_{11}\right| \mathrm{dB}$ for the rectangle shaped PIFA indicates a $900 \mathrm{MHz}$ resonance.

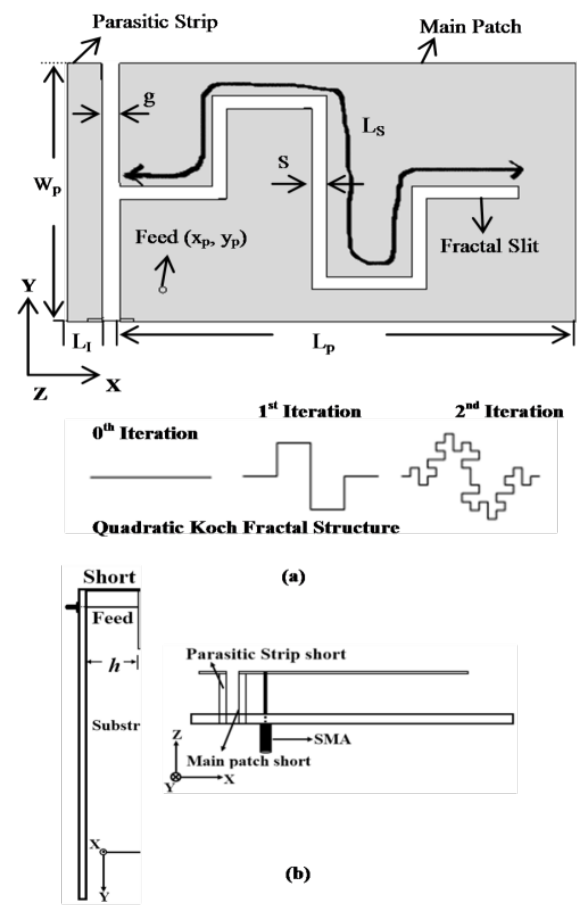

Figure 1: Geometry of the proposed antenna (a) top view and (b) side views.

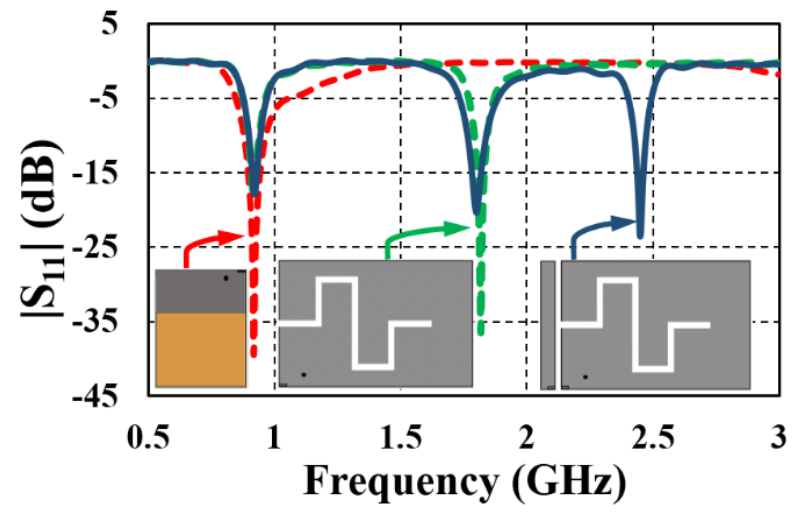

Figure 2: $\left|\mathrm{S}_{11}\right| \mathrm{dB}$ variation versus frequency for initial PIFA design, with and without fractal slit, and along with a parasitic strip.

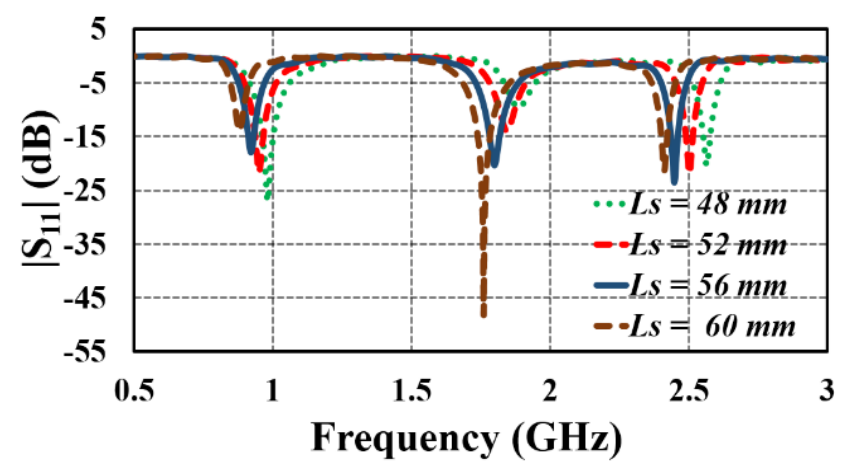

Figure 3: $\left|\mathrm{S}_{11}\right| \mathrm{dB}$ curve variation versus frequency for different total slit length $\left(L_{s}\right)$

\subsection{Dual-band PIFA with fractal shaped slit}

On the designed PIFA, a slit is implemented to introduce a plurarism in antenna current distribution profile that translates into multifrequency resonances. Implementing slit in the radiating patch facilitates multiband operation which includes $1800 \mathrm{MHz}$ (DCS band) frquency band. Fractal geometry has advantages of self similarity, space filling and larger perimeter within the fixed surface area so we choose to implement quadratic Koch fractal shaped slit. A quadratic Koch slit of slit length $\left(L_{s}\right) 56 \mathrm{~mm}$ and $1.00 \mathrm{~mm}$ slit width $(s)$, has been etched and tunned using CST Microwave Studio $2011^{\mathrm{TM}}$ as shown in Figure. 1. Optimization and simulation results in a dual- band PIFA structure having compact volume of $32 \times 20 \times 8 \mathrm{~mm}^{3}$ resonating at GSM $(900 \mathrm{MHz})$ and DCS band $(1800 \mathrm{MHz})$.The DCS (1800 $\mathrm{MHz}$ ) band can be excited by lengthening the resonant path to $42 \mathrm{~mm}$ by slit implementation, which is about-one quarter of the wavelength at $1800 \mathrm{MHz}$. Fig. 2 clearly shows the addition of resonance frequency with the introduction ofthe slit. The $\left|\mathrm{S}_{11}\right| \mathrm{dB}$ variation with frequency for different fractal slit length $\left(L_{S}\right)$ is shown in Fig. 3 and variation of resonance for differnet slit width $(s)$ is shown in Fig. 4, best 
impedance matching for the desired frecuency is shown as curve indicated with solid line for $L_{s}=56 \mathrm{~mm}$ and $s=$ $1.0 \mathrm{~mm}$.

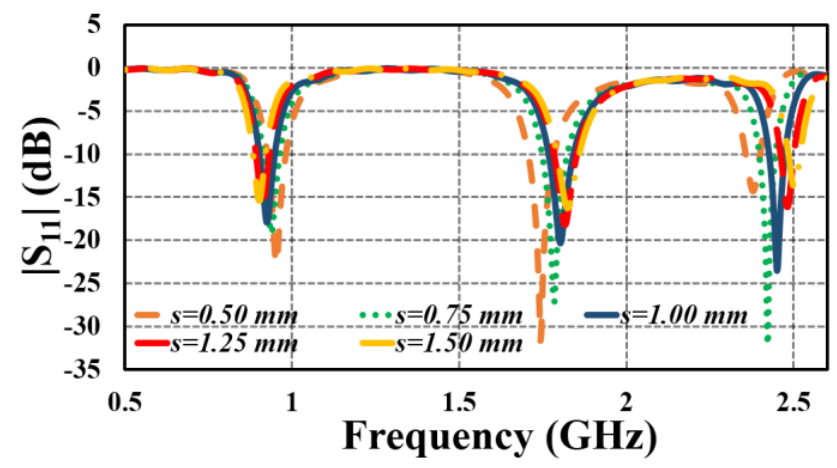

Figure 4: $\left|\mathrm{S}_{11}\right| \mathrm{dB}$ variation versus frequency for different slit width $(s)$.

\subsection{Inclusion of parasitic patch for introducing the third band in PIFA}

A parasitic patch is added to finally obtain a tri-band PIFA with resonance frequency at WLAN (IEEE 802.11b) band $2.45 \mathrm{GHz}$. Parasitic patch of dimension $20 \mathrm{~mm} \times 2.4 \mathrm{~mm}$ is added parellel to the radiating patch, this is obtained by using (1) for the corresponding resonance frequency of 2.45 GHz. Hence, the total length of current path, i.e, $(20+2.4+$ $8 \mathrm{~mm}=30.4 \mathrm{~mm}$ ) which is approximately $0.25 \lambda$ corresponding to the resonance frequency of $2.45 \mathrm{GHz}$. There is a coupling beteween main patch and parasitic patch, so it is placed at a balanced coupled gap $(g)$ of $1.25 \mathrm{~mm}$ from the main patch since it gives the best impedance matching at $2.45 \mathrm{GHz}$.The addition of third resonance frequency by adding parasitic patch can be observed clearly in Fig. 2. A compact PIFA structure is obtained for triple band application having overall optimized dimension $\left(\mathrm{L}_{\mathrm{p}}+\mathrm{L}_{\mathrm{i}}\right) \times \mathrm{W}_{\mathrm{p}} \times \mathrm{h}$ of $35.65 \mathrm{~mm} \times 20 \mathrm{~mm} \times 8 \mathrm{~mm}$, with a fractal slit of peripheral length $\left(L_{s}\right) 56 \mathrm{~mm}$. As indicated in Fig. 1, the proposed antenna is fed by $50 \Omega$ SMA connector at position $\left(x_{p}, y_{p}\right)=(3 \mathrm{~mm}, 2.5 \mathrm{~mm})$ on the main patch. The influence of the gap $(g)$ on resonance characteristics of the tri-band PIFA is given in Fig. 5. The third mode resonating frequency decreases with increasing the gap as coupling gap capacitance increases with the gap values. The antenna height parametric analysis is shown in Fig. 6. In figure 6, it is seen that substrate height has effect on resonance frequency at third band but has no effect on first band. It is due to the value of $L / h$ ratio of parasitic patch, which is $<1$. So, with increase in $\mathrm{h}$ the fringing effect is also increased which decreases the resonance frequency at third mode.Also increase in height lengthens the resonant path, i.e, decreases the resonance frequency. The final optimized value of $8 \mathrm{~mm}$ for height is used in design.

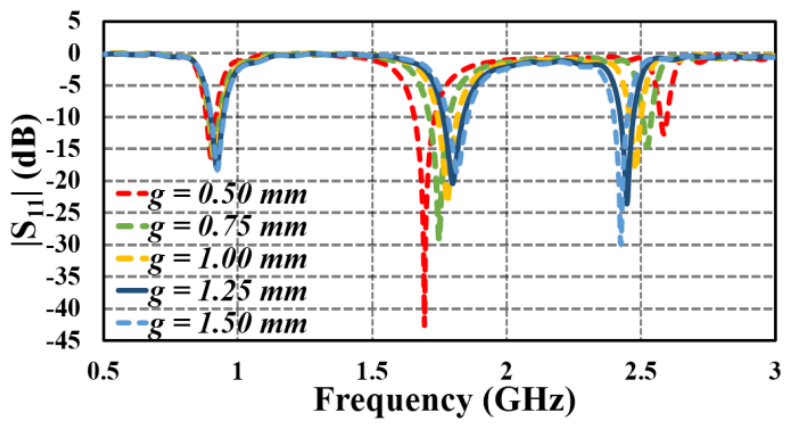

Figure 5: $\left|\mathrm{S}_{11}\right| \mathrm{dB}$ variation versus frequency for different gap between main patch and parasitic patch $(g)$.

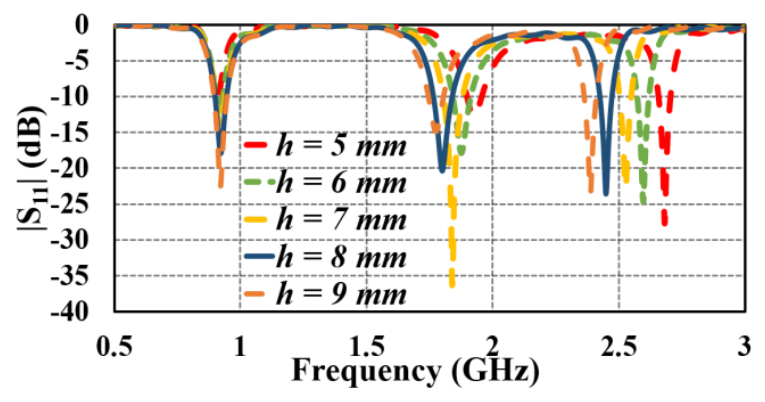

Figure 6: $\left|\mathrm{S}_{11}\right| \mathrm{dB}$ variation versus frequency for different antenna height $(h)$.

\section{Results and discussion}

In order to verify the performance of the proposed antenna design a prototype is developed and measured using $\mathrm{R} \& \mathrm{~S}$ ZVA 40 vector network analyzer. Fig. 7 plots the simulated and measured $\left|S_{11}\right| d B$ curve of the proposed antenna. Acceptable agreement has been achieved between the simulated and measured results. The $-6 \mathrm{~dB}$ (3:1 VSWR) measured frequency range at each band is obtained as 880964, 1741-1873 and 2407-2488 MHz, which corresponds to $9.12 \%, 7.33 \%$ and $3.30 \%$ impedance bandwidth, respectively.

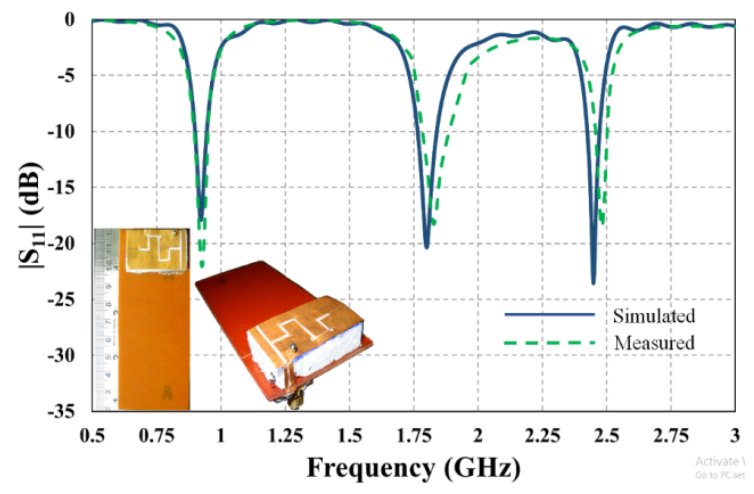

Figure 7: Measured and simulated $\left|\mathrm{S}_{11}\right| \mathrm{dB}$ of the antenna. $\left(L_{s}=56 \mathrm{~mm}, g=1.25 \mathrm{~mm}\right.$, parasitic patch $\mathrm{size}=20 \mathrm{~mm} \times 2.4 \mathrm{~mm}$ ) . 


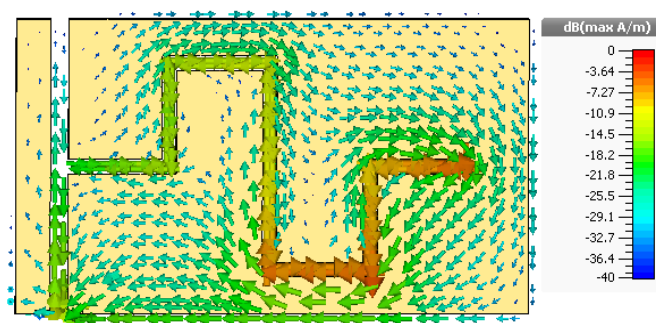

(a)

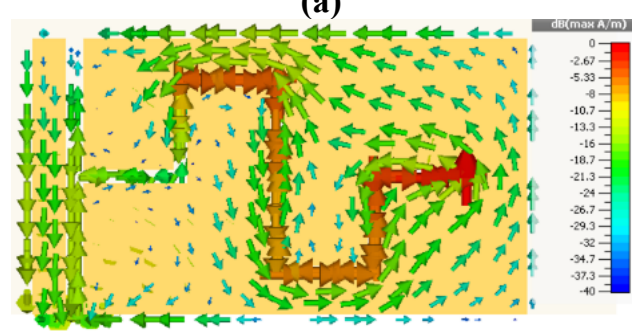

(b)

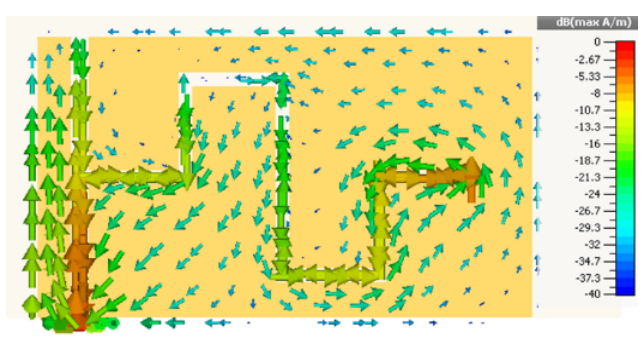

(c)

Figure 8: Illustration of current distribution on the proposed PIFA at (a) $0.9 \mathrm{GHz}$, (b) $1.8 \mathrm{GHz}$ and (c) 2.45 $\mathrm{GHz}$.

To have a better insight into the tri-band resonance behaviour of the proposed antenna, the surface current distribution of the proposed antenna at $0.9 \mathrm{GHz}, 1.8 \mathrm{GHz}$ and $2.45 \mathrm{GHz}$ is shown in Fig. 8. At first resonating mode, a quarter wavelength resonance at $0.9 \mathrm{GHz}$ is excited with current null which is occurred in the main patch. At $1.8 \mathrm{GHz}$ resonant mode, current maxima is seen to be happened in the Koch fractal slit having a resonant path corresponding to the quarter wavelength at $1.8 \mathrm{GHz}$ DSC band and a halfwavelength resonance at $2.45 \mathrm{GHz}$ is excited with the maximum current distribution on the face of parasitic patch for WLAN(IEEE 802.11b) band, where another current maxima has been occurred on the slit end.

The measured and simulated radiation patterns of the antenna are shows in Fig. 9, Fig.10 and Fig. 11 at three representative frequencies of $0.92,1.8$ and $2.44 \mathrm{GHz}$, respectively. At each frequency, normalized radiation patterns in three principal planes are shown. The measured antenna gain are $2.44,4.48$ and $3.86 \mathrm{dBi}$ at $0.92,1.8$ and
$2.44 \mathrm{GHz}$, respectively. The overall volume of the antenna is $35.65 \times 20 \times 8 \mathrm{~mm}^{3}$, which is about $24.5 \%$ less than the initial PIFA structure volume. All the results shows that this antenna can be employed for GSM900/DCS1800/WLAN(IEEE 802.11b) mobile phone applications.
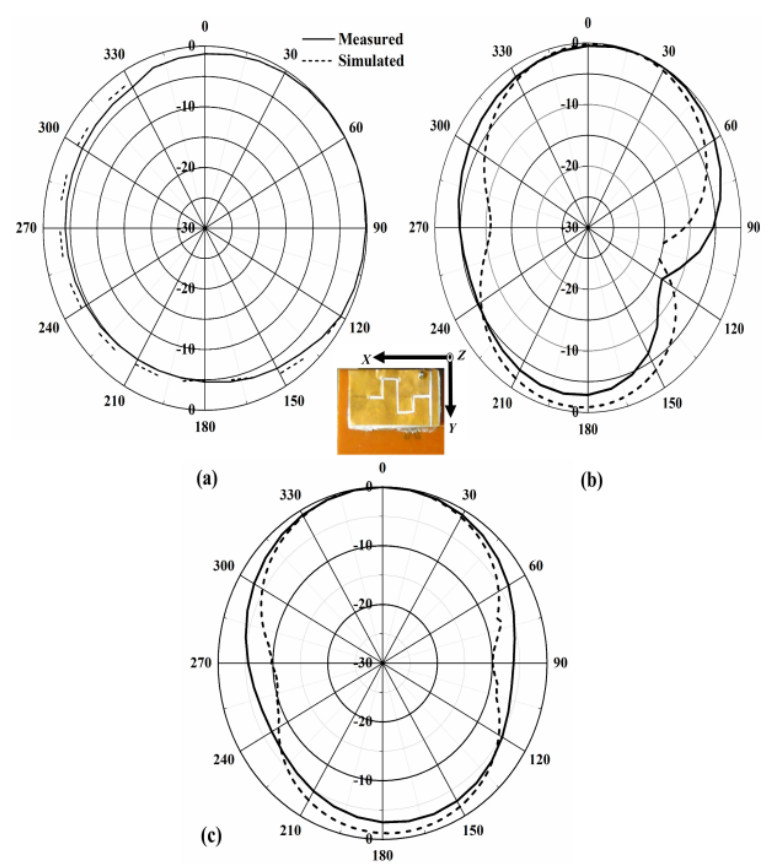

(b)

Figure 9: Measured and simulated 2-D principal plane pattern of the proposed antenna at $0.9 \mathrm{GHz}$ (a) $x-z$ plane (b) $y-z$ plane (c) $x-y$ plane.
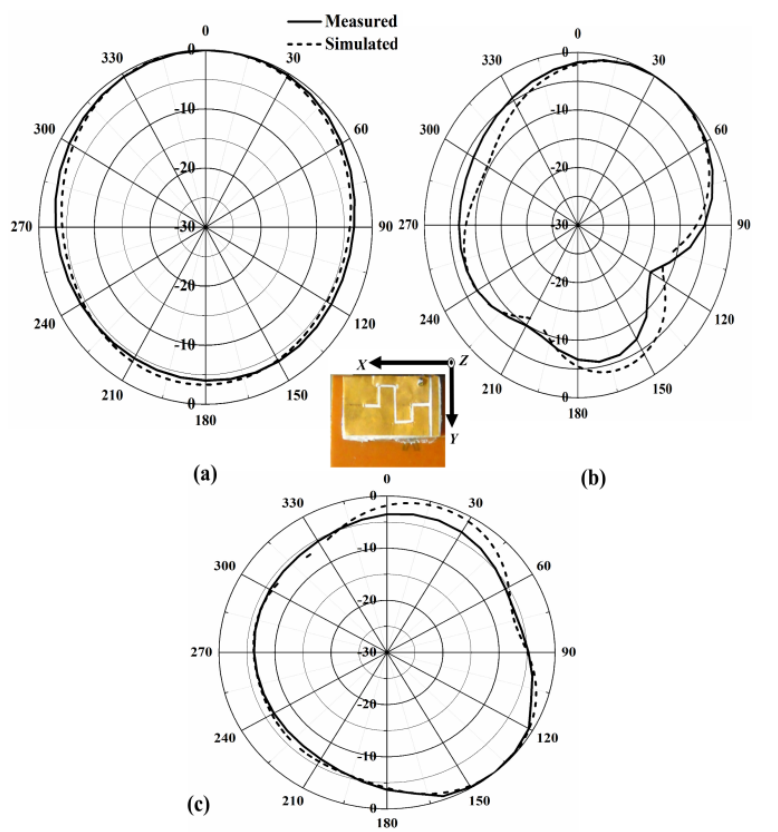

(b)

Figure 10: Measured and simulated 2-D principal plane pattern of the proposed antenna at $1.8 \mathrm{GHz}$ (a) $x-z$ plane (b) $y$-z plane (c) $x-y$ plane. 


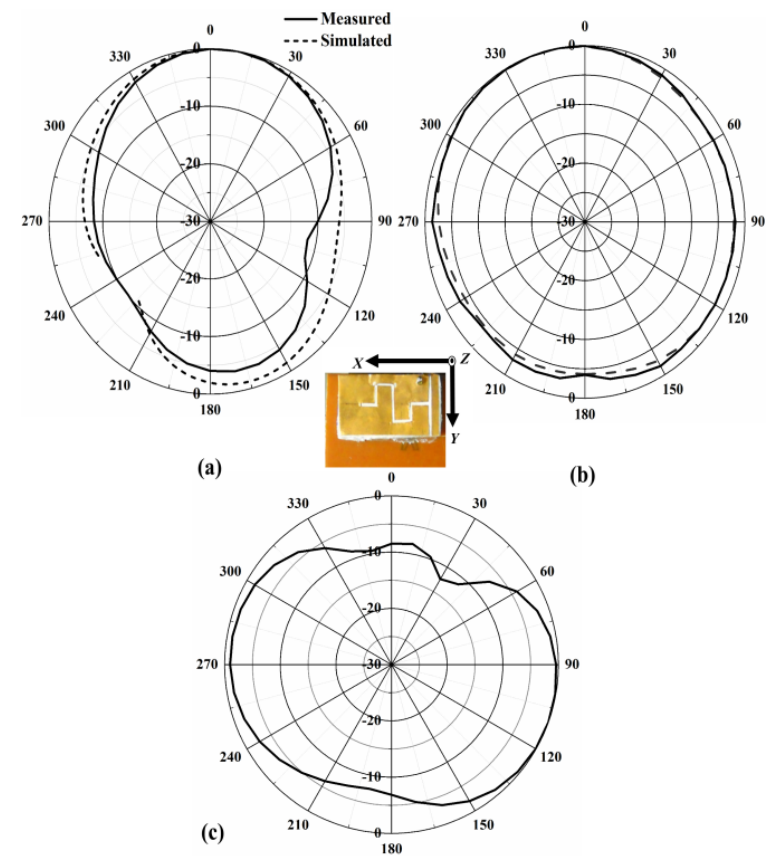

Figure 11: Measured and simulated 2-D principal plane pattern of the proposed antenna at $2.45 \mathrm{GHz}$ (a) $x-z$ plane (b) $y-z$ plane (c) $x-y$ plane.

\section{Conclusion}

A compact tri-band PIFA is proposed and studied for the application in mobile handsets. Fractal slit is implemented on the main radiating element for dual-band operation. With the addition of a parasitic patch at an optimum distance from main patch, a third frequency band is obtained. The creation of the slit first reduces the dimension of the PIFA due to reduced capacitance and again with the inclusion of the parasitic strip, that cases capacitive loading results in enhancement of overall size in order to tune the resonances to usable frequencies. The overall antenna dimension is $35.65 \mathrm{~mm} \times 20 \mathrm{~mm}$ with a height of $8 \mathrm{~mm}$ above the ground plane. The measured antenna resonances are obtained at the $0.92 \mathrm{GHz}, 1.8 \mathrm{GHz}$ and $2.44 \mathrm{GHz}$. The proposed antenna has been designed and fabricated. The radiation characteristics have been observed which ensure its suitability for cellular communication.

\section{References}

[1] Virga L.K.; Samii Y. R.: Low-profile EnhancedBandwidth PIFA Antennas for Wireless Communicationspackaging, IEEE Transactions on Microwave Theory and Techniques, vol. 45, pp. 1879$1888,1997$.

[2] LiuZ. D.;Hall P. S.; Wake D.,:Dual-Frequency Planar Inverted-F antenna, IEEE Transactionson Antenna and Propagation, vol.45,pp. 1451-1458,1997.

[3] Rowell C. R. and Murch R. D.,: A Compact PIFA Suitable for Dual-Frequency 900/1800-MHz Operation,
IEEE Transactions on Antennas and Propagation, vol.46, pp. 596-598, 1998.

[4] Salonen P.;KeskilammiM.and Kivikoski M.,: Single Feed Dual-Band Planar Inverted-F Antenna with UShaped Slot, IEEE Transactions on Antennas and Propagation, vol.48, pp. 1262-1264,2000.

[5] Kwon Y.;Moon J.; Park S.,: An Internal Triple-Band Planar Inverted-F Antenna,IEEE Antennas and Wireless Propagation Letters, vol. 2, pp. 341-344,2003.

[6] Shin Y.-S.;Kim B.-N.;Kwak W.-II;Park S.-O.,: GSM/DCS/IMT-200 Triple-Band Built-In Antenna for Wireless Terminals, IEEE Antennas and Wireless Propagation Letters, vol. 3, pp. 104-107,2004.

[7] Row J.-S.;: Dual-frequency Triangular Planar InvertedF Antenna, IEEE Transactions on Antenna and Propagation, vol. 53, pp. 874-876,2005.

[8] Kwak W.-II; Park S.-O; Kim J.-S,: A Folded Planar Inverted-F AntennaforGSM/DCS/Bluetooth TripleBand Application,IEEE Antennas and Wireless Propagation Letters, vol. 5, pp. 18-21,2006.

[9] Wong K.-Lu;Lin Y.-C.; Ting-Chih T., Thin Internal GSM/DCS Patch Antenna for a Portable Mobile Terminal, IEEE Transactions on Antenna and Propagation, vol. 54,pp. 238-242, 2006.

[10] Azad M. Z.;Ali M.,: A Miniaturized Hilbert PIFA for Dual-PIFA Mobile Wireless Applications,IEEE Antennas and Wireless Propagation Letters, vol.4, pp.59-62, 2005.

[11] Cho Y. J.;Hwang S. Ho;Park S. -Ook,: A Dual-Band Internal Antenna With a Parasitic Patch for Mobile handsets and the Consideration of the Handset Case and Battery,IEEE Antennas and Wireless Propagation Letters, vol. 4, pp. 429-432, 2005.

[12] Kim D.-Y.;Lee J. W.;Cho C. S.; Lee T. K.,: Design of a Compact Tri-Band PIFA Based on Independent Control of the Resonant Frequencies,IEEE Transaction On Antennas And Propagation, vol. 56, pp. 1428-1435, 2008.

[13] Wang H.;Zheng M.,: An internal triple-Band WLAN Antenna,IEEE Antennas and Wireless Propagation Letters, vol.10, pp. 569-572, 2011.

[14] Lin K.-C.;Lin C.-H.;Lin Y.-C.,: Simple Printed Multiband Antenna With Novel Parasitic-Element Design for Multistandard Mobile Phone Applications, IEEE Transactions on Antenna and Propagation, vol.61, pp.488-491, 2013.

[15] Lee C. T.; Su, S. W.,: Very-low-profile, 2.4/5.2/5.8-GHz, triband WLAN antenna for laptoptablet computer with complete metal cover, Microwave and Optical Technology Letters, vol.58, 2016.

[16] Wakrim L.; Ibnyaich S.; HassaniM. M.,: Multiband operation and performance enhancement of the PIFA antenna by using particle swarm optimization and overlapping method, Applied Computational Intelligence and Soft Computing, 2017, doi.org/10.1155/2017/3481709. 